\title{
E2F8 promotes hepatic steatosis through FABP3 expression in diet-induced obesity in zebrafish
}

Yasuhito Shimada ${ }^{1,2,3,4,5}$, Shisei Kuninaga ${ }^{6}$, Michiko Ariyoshi ${ }^{1}$, Beibei Zhang ${ }^{1}$, Yasuhiko Shiina ${ }^{6}$, Yoshinori Takahashi $^{6}$, Noriko Umemoto ${ }^{1,2}$, Yuhei Nishimura ${ }^{1,2,3,4,5}$, Hiroyuki Enari ${ }^{6}$ and Toshio Tanaka ${ }^{1,2,3,4,5^{*}}$

\begin{abstract}
Background: Diet-induced hepatic steatosis is highly associated with nonalcoholic fatty liver disease, which is related to the development of metabolic syndrome. While advanced stage nonalcoholic hepatic steatosis and steatohepatitis (NASH) result ultimately in fibrosis and cirrhosis, the molecular basis for lipid droplet formation is poorly understood. Common pathways underlie the pathology of mammalian obesity and the zebrafish diet-induced obesity model (DIO-zebrafish) used in this study.

Methods: Our analysis involved a combination of transcriptome (DNA microarray) and proteome (two-dimensional electrophoresis) methods using liver tissue from DIO-zebrafish to find candidate genes involved in hepatic steatosis. We conducted intraperitoneal injection (i.p.) of morpholino antisense oligonucleotides (MOs) for each gene into DIO-zebrafish. We also conducted in vitro overexpression in human cells. Additionally, we examined gene expression during feeding experiments involving anti-obesity compounds, creatine and anserine.

Results: We found that fatty acid binding protein 3 (fabp3) and E2F transcription factors were upregulated in hepatic steatosis. E2f8 MO i.p. suppressed fabp3 expression in liver, and ameliorated hepatic steatosis. In human cells (HepG2), E2F8 overexpression promoted FABP3 expression. Additionally, CO-administration of creatine and anserine suppressed obesity associated phenotypes including hepatic steatosis as indicated by e2f8 and fabp3 down regulation.

Conclusion: We discovered that the e2f8-fabp3 axis is important in the promotion of hepatic steatosis in DIO-zebrafish. The combination of transcriptome and proteome analyses using the disease model zebrafish allow identification of novel pathways involved in human diseases.
\end{abstract}

Keywords: Fatty liver, Obesity, Zebrafish, High-fat feeding, DNA microarray, Two-dimensional electrophoresis, Cross-species analysis

\section{Background}

Hepatic steatosis is an accumulation of fat in the liver, and causes of non-alcoholic fatty liver disease (NAFLD) encompass a spectrum of diseases ranging from steatohepatitis (NASH) to increasing fibrosis and eventual cirrhosis [1], sometimes subsequent to hepatocellular carcinoma [2]. This is associated with metabolic syndrome, especially obesity, hyperlipidaemia and diabetes

\footnotetext{
* Correspondence: tanaka@doc.medic.mie-u.ac.jp

'Department of Molecular and Cellular Pharmacology, Pharmacogenomics and Pharmacoinformatics, Mie University Graduate School of Medicine, 2-174, Edobashi, Tsu, Mie, Japan

${ }^{2}$ Department of Systems Pharmacology, Mie University Graduate School of Medicine, Mie, Japan

Full list of author information is available at the end of the article
}

[3], and is now the most common liver disease in both adults and children worldwide [4]. The estimated prevalence of NAFLD in the general population ranges from 5 to $20 \%$ and up to $75 \%$ of patients with obesity and diabetes mellitus [5]. Elevated plasma concentrations of glucose and fatty acids promote hepatic fatty acid synthesis and/or impair $\beta$-oxidation leading to hepatic steatosis [6]. Lipid accumulation in the liver is also linked with the progression of endoplasmic reticulum and mitochondria stress, and impaired autophagy, resulting in the condition known as lipotoxicity [7]. Insulin resistance is one of the most important factors in the development of hepatic steatosis, however, pharmacological treatment related to insulin-sensitizing agents (metformin 
and thiazolidinediones [TZDs]) has been studied in hepatic steatosis with conflicting results in clinical situations [8]. This suggests that the primary molecular basis for formation of hepatic steatosis is not related to insulin resistance. In fact, many genes implicated in hepatic steatosis are not related to insulin signalling, including FABPs. Dysregulated FABPs have been associated with a number of diseases, including obesity and NAFLD [9]. Preventing hepatic steatosis is critical for blocking the initial stages of NAFLD. Although the therapeutic mechanisms and gene targets involved in the accumulation of triglycerides (TG) in the liver and subsequent hepatocellular damage are not fully characterized, it is thought the process involves conventional lipid metabolism with insulin resistance.

The zebrafish (Danio rerio), a small vertebrate, has recently emerged as a genetically tractable model animal for human diseases [10]. Zebrafish have multiple advantages, such as a high degree of genetic conservation compared with mammals, their ease of genetic manipulation, and large clutch sizes making them amenable to high-throughput screening, including behaviour analysis [11]. In addition, lipid metabolism in zebrafish is similar to humans in that absorption occurs through the intestine with the aid of bile produced in the liver [12], transport of cholesterol is mediated by lipoproteins [13], and TG is stored in visceral, subcutaneous, and intramuscular adipocyte deposits [14]. In light of these similarities to mammals and the advantages of the system, the zebrafish model has been used in the field of lipid metabolism research for studies of lipid-related diseases, including dyslipidaemia with hepatic steatosis induced by diet-induced obesity (DIO) $[15,16]$, atherosclerosisinduced by high-cholesterol diet [17] and in a transgenic model of obesity [14]. In our previous studies, transcriptome analyses showed that the common pathways in hepatic steatosis of DIO-zebrafish were highly consistent with human obesity and rodent models of DIO [16]. We have also clarified the anti-hepatic steatosis mechanism of the bioactive molecule, eriocitrin [18], using the DIOzebrafish.

In this study, we discovered that the fatty acid binding protein 3 (fabp3) gene was involved in hepatic steatosis by using DNA microarray and two-dimensional electrophoresis (2-DE) analyses of DIO-zebrafish, and the knockdown of e2f transcription factor 8 (e2f8) suppresses the development of hepatic steatosis via downregulation of fabp3 in DIO-zebrafish.

\section{Methods}

\section{Ethics statement}

This study has been approved by the Ethics Committee of Mie University, and was performed according to Japanese animal welfare regulations outlined in the 'Act on Welfare and Management of Animals' (Ministry of Environment of Japan) and complied with international guidelines. After the experiments, the fish were sacrificed by an overdose of anaesthetic solution, tricaine methanesulfonate $(500 \mathrm{mg} / \mathrm{L}$; Sigma-Aldrich, St. Louis, MO, USA), in system water buffered with sodium bicarbonate (0.7 g/litter; Wako Pure Chemicals, Osaka, Japan).

\section{DIO-zebrafish experiments}

The zebrafish $A B$ wild type was supplied by the Zebrafish International Resource Center (University of Oregon, Eugene, OR) and maintained in our facility according to the established protocols [19]. To induce DIO, zebrafish were assigned into each dietary group with five fish per 1.7-L tank. From 3-4 months post fertilization (mpf), zebrafish in the overfeeding group were fed three times per day with Hikari Labo M-450 (HL450; Kyorin, Hyogo, Japan) containing beef tallow (HL450-BT; $7 \%$ weight volume/fish weight/day) as high-fat (HF) diet. The normal feeding (NF) group was also fed one time per day with HL450 (3\% weight volume/fish weight/day). Each group contained 10 zebrafish. Every week we measured the body weight and calibrated the feeding volume daily. For HL450-BT, we prepared HL450 with $15 \%$ total fat with beef tallow (Wako Pure Chemicals). Nutrition facts and the fatty acid compositions of HL450 and HL450-BT were described in Table 1 and Additional file 1: Table S1. Anserine (L-anserine nitrate salt; Sigma-Aldrich) and creatine (creatine anhydrous; Wako Pure Chemicals) were added to HL450-BT (1.34 mg/gBW/day and $0.14 \mathrm{mg} / \mathrm{gBW} /$ day, respectively), according to our previous method [20]. Zebrafish were fed to satiation three times daily. Satiation was defined as the point within a $5 \mathrm{~min}$, where zebrafish were no longer actively searching for food [21]. Food consumption was calculated as the difference between the weight of food offered and food remaining.

\section{Measurement of zebrafish body weight, length, BMI, plasma TG and fasting blood glucose}

The body weight and length of zebrafish were measured weekly throughout the study as described previously [15]. The body mass index (BMI) was calculated by

\begin{tabular}{lll}
\multicolumn{2}{l}{ Table 1 Nutrition information of fish foods } \\
\hline Nutrition information Per container (100 g) & HL450 & HL450-BT \\
\hline Energy (kcal) & 379 & 405 \\
Water (g) & 7.4 & 7.5 \\
Protein (g) & 41.7 & 40.0 \\
Lipid (g) & 9.8 & 14.6 \\
Carbohydrate (g) & 31.0 & 28.4 \\
Ash (g) & 10.1 & 9.5
\end{tabular}


dividing the body weight ( $\mathrm{g}$ ) by the square of the body length $(\mathrm{cm})$. For the blood chemistry analyses, zebrafish were deprived of food overnight and blood was withdrawn from the dorsal artery by a heparinized glass capillary needle (GD-1; Narishige, Tokyo, Japan) at the end of the feeding experiment. Fasting blood glucose and plasma TG were measured as described previously [22].

\section{Oil Red O staining}

Liver tissues were collected from zebrafish by surgical manipulation under a MZ16F stereoscopic microscope (Leica Microsystems, Wetzlar, Germany). The preparation of liver sections and Oil Red O staining were performed as described previously [23]. Sections were also counterstained with Mayer's hematoxylin (Wako Pure Chemicals, Osaka, Japan) to visualize the nuclei according to the manufacturer's protocol. Lipid accumulation was quantified using the WinROOF version 5 (Mitani, Fukui, Japan).

\section{Measurement of liver lipid in zebrafish}

Hepatic lipids were extracted from fixed liver tissues as described previously [24]. The fixation was conducted with RNAlater (Life Technologies) for at least 3-month at $4{ }^{\circ} \mathrm{C}$. The dried lipid residues were dissolved in $15 \mu \mathrm{L}$ of cyclohexane. Hepatic lipids were measured using the Lipid Quantification Kit (Colorimetric; Cell Biolabs, San Diego, CA, USA) according to the manufacturer's protocol.

\section{Zebrafish DNA microarray}

The samples for each condition were obtained from four independent experiments. DNA microarray experiments were conducted as described previously [16]. In brief, total RNA was extracted according to the protocol for Isogen (Nippon Gene, Tokyo, Japan), in combination with the clean-up protocol of the RNeasy Mini Kit (Qiagen, Hilden, Germany). The DNA microarray experiments were conducted using the Low RNA Input Fluorescent Linear Amplification Kit and G2518A Agilent Zebrafish Whole Genome Oligo Microarrays (Agilent Technologies, Santa Clara, CA, USA). The hybridized microarrays were scanned using an Agilent G2565BA microarray scanner and quantified using Feature Extraction software (Agilent Technologies). The data were further analysed using GeneSpring GX10 software (Agilent Technologies) to identify differentially expressed genes between two groups $(P<0.05)$. The probes were converted to human orthologs using the Life Science Knowledge Bank (World Fusion, Tokyo, Japan). Sub-network enrichment analysis (SNEA) [25] was conducted using Pathway Studio version 9 (Elsevier, Amsterdam, Netherlands).

\section{Zebrafish 2-DE}

Liver tissues from three adult zebrafish were dissected and pooled in liquid nitrogen. The frozen tissues were then homogenized in ice-cold $85 \%$ methanol. The homogenates were centrifuged at $12,000 \times \mathrm{g}$ for $20 \mathrm{~min}$ at $4{ }^{\circ} \mathrm{C}$. The pellets were dissolved in lysis buffer $(8 \mathrm{M}$ urea, $2 \mathrm{M}$ thiourea, $10 \%$ isopropanol, $0.1 \%$ Triton X-100, $4 \%$ 3-[(3-cholamidopropyl) dimethylammonio]-1 propanesulfonate, and $50 \mathrm{mM}$ dithiothreitol [DTT]), and were shaken for $40 \mathrm{~min}$ at room temperature. After precipitation $\left(12,000 \times \mathrm{g}\right.$ for $40 \mathrm{~min}$ at $\left.20^{\circ} \mathrm{C}\right)$, the protein concentration was estimated by Bradford assay (Bio-Rad, Richmond, CA, USA), and isoelectric focusing was performed on a 11-cm-long 3-10 nonlinear pI range IPG strip (Bio-Rad) using $200 \mu \mathrm{g}$ of total liver protein (in $200 \mu \mathrm{L}$ lysis buffer). Following a 4-h passive rehydration, isoelectric focusing was performed at $20{ }^{\circ} \mathrm{C}$ and $50 \mu \mathrm{A}$ with the following four-step gradient program: $30 \mathrm{~V}$ for $10 \mathrm{~h}, 200 \mathrm{~V}$ for $1 \mathrm{~h}, 500 \mathrm{~V}$ for $1 \mathrm{~h}, 1000 \mathrm{~V}$ for $1 \mathrm{~h}$, $8000 \mathrm{~V}$ for half an hour. The strips were then equilibrated in buffer I (6 M urea, 62.4 mM Tris [pH 6.8], $2 \%$ sodium dodecyl sulphate, $4 \%$ glycerol, $50 \mathrm{mM}$ DTT, and $0.01 \%$ bromophenol blue). Two-dimensional electrophoresis was performed in $12.5 \%$ sodium dodecyl sulphate-polyacrylamide gel $(11 \times 11 \mathrm{~cm})$ electrophoresis for $2 \mathrm{~h}$ at $40 \mathrm{~mA}$. The gels were then fixed and stained (0.0028 \% Coomassie Brilliant Blue R-250 and $28 \%$ isopropanol) for $3 \mathrm{~h}$ and de-stained with $10 \%$ acetic acid. The gels were scanned and documented using a GS-800 Calibrated Densitometer (Bio-Rad) and analysed using PD Quest 2-DE Analysis Software (Bio-Rad).

\section{Peptide mass fingerprinting}

The protein band was cut and then transferred to a microtube loaded with $100 \mu \mathrm{l}$ of $50 \%$ acetonitrile containing Tris-HCl. Gel pieces were dehydrated twice with this $50 \%$ ACN solution. The dried gel particles were rehydrated at $37{ }^{\circ} \mathrm{C}$ for $15 \mathrm{~h}$ using an XL-TrypKit (APRO Science, Tokushima, Japan) according to the manufacturer's instruction. After trypsin digestion, the proteins were examined using a matrix-assisted laser desorption ionization time of flight mass spectrometer (Bruker Daltonics, MA, USA). The obtained peptide mass fingerprinting data were screened for preliminary protein IDs using GPS Explorer software version 3.6 (Applied Biosystems, Foster City, CA, USA) against the Danio rerio NCBI database (2008) and Swiss-Prot 2009 (MASCOT version 2.0; Matrix Science, Boston, MA, USA).

\section{Intraperitoneal administration of morpholinos}

Morpholinos (MOs) were designed and synthesized by Gene Tools LLC (Philomath, OR, USA). The MO 
sequences are shown in Additional file 2: Table S2. For the negative control groups, the control MO (human $\beta$-globin mutant sequence; GeneTools) was used. Intraperitoneal (i.p.) administration of morpholinos was conducted as previously described [26]. In detail, $2 \mu \mathrm{l}$ samples of each $\mathrm{MO}$ solution were diluted by adding $3 \mu \mathrm{l}$ of OPTI-MEM I (Life Technologies), which was combined with a Lipofectamine 2000 (Life Technologies) mixture $(2 \mu \mathrm{l}$ of Lipofectamine 2000 and $3 \mu \mathrm{l}$ of OPTIMEM I). The MO mixtures were incubated at room temperature for $20 \mathrm{~min}$ and injected into the abdominal cavity of 3-4 mpf zebrafish (approximately $50 \mu \mathrm{mol} / \mathrm{kg}$ body weight) using FemtoJet (Eppendorf, Hamburg, Germany) with a fine-polished GD-1 glass capillary (Narishige, Tokyo, Japan). Intraperitoneal administration was conducted once a week during feeding experiments, starting from 1 week before the feeding experiment.

\section{Western blot}

The liver tissues of DIO-zebrafish were collected by surgical extraction. Lysate protein was prepared by homogenization and sonication in T-PER Tissue Protein Extraction Reagent (Thermo Scientific, Rockford, IL) with protease inhibitor cocktail (Thermo Scientific). The samples were centrifuged at $12,000 \times \mathrm{g}$ for $30 \mathrm{~min}$ after homogenization with the MM300 Mixer Mill $(30 \mathrm{~Hz}$ for 2 min; Retsch, Haan, Germany). For western blot analysis, protein samples were separated by 4-15\% SDSPAGE and transferred onto a polyvinylidene fluoride (PVDF) membrane (Bio-Rad), and blocked at $20^{\circ} \mathrm{C}$ with TBS containing $5 \%$ skim milk (Becton, Dickinson and Company, Sparks, MD, USA) for $90 \mathrm{~min}$. The membrane was incubated with goat polyclonal to E2F8 (1:2000; Aviva Systems Biology, San Diego, CA, USA) at $4{ }^{\circ} \mathrm{C}$ for $16 \mathrm{~h}$, and then washed with TBS containing $0.05 \%$ Tween-20 (TBST) five times. Horseradish peroxidase (HRP) -conjugated rabbit anti-goat (1:5000; Santa Cruz Biotechnology, Santa Cruz, CA, USA) secondary antibodies were used to detect E2F8. After five washes with PBST, immunoreactions were detected using TMB stabilized substrate for HRP (Promega, WI, USA) with a Molecular Imager Chemi Doc XRS Plus (Bio-Rad), and analysed using PD Quest Advanced/Basic Ver.8.0 (Bio-Rad).

\section{Cell culture and transfection}

HepG2 human hepatocarcinoma cells were cultured in Dulbecco's modified Eagle's medium (Life Technologies, Carlsbad, CA, USA), supplemented with $100 \mu \mathrm{g} / \mathrm{ml}$ streptomycin sulphate (Sigma-Aldrich, St. Louis, MO, USA), $100 \mathrm{U} / \mathrm{ml}$ penicillin G (Sigma-Aldrich) and $10 \%(\mathrm{v} / \mathrm{v})$ foetal bovine serum (Life Technologies), and maintained at $37{ }^{\circ} \mathrm{C}$ in an atmosphere of $5 \% \mathrm{CO}_{2}$ and
$95 \%$ air. Cells were transfected at $70 \%$ confluence with $2 \mu \mathrm{g}$ plasmid DNA by using Lipofectamine 2000 reagent (Life Technologies). Plasmids containing human E2F3 (FHC12471) and E2F8 (FHC24174) were purchased from Promega (Madison, WI, USA). HaloTag Control Vector was used as a control for transfection. To confirm transfection efficacy, HaloTag TMR Ligand (Promega) was used according to the manufacturer's protocol.

\section{qPCR}

For liver tissues of adult zebrafish, total RNA of each sample was purified as described previously [27]. For cultured cells, total RNA was also purified using the RNeasy Mini Kit. First-strand cDNA was prepared with 200 ng total RNA using the Super Script III First-strand System (Life Technologies) with random primers (Life Technologies). qPCR was performed with Power SYBR Green Master Mix (Applied Biosystems) in triplicate, according to the manufacturer's protocol. The sequences of the primers are shown in Additional file 3: Table S3. The oligonucleotides of these primers were synthesized by Life Technologies.

\section{Statistical analysis}

All data were represented as mean \pm SEM. Differences between the two groups were examined for statistical significance using Student's $t$ test. For multiple comparisons, we used one-way analysis of variance followed by Bonferroni-Dunn multiple comparison. $P<0.05$ was considered to denote statistical significance.

\section{Results}

Proteome and transcriptome analyses of DIO-zebrafish

Typical image of DIO-zebrafish was shown in Fig. 1a. HF significantly $(P<0.05)$ increased body weight after 7 days of feeding (Fig. 1b). Body length was also increased by HF (Fig. 1c), while the increased rate was less than that for body weight (1.06-fold vs. 1.5-fold on day 21). Body mass index (BMI) was also significantly $(P<0.05)$ increased after 7 -day feeding experiments (Fig. 1d). The plasma TG was increased significantly $(P<0.05$, Fig. 1e) on day 21 , however fasting glucose was not affected by HF (Fig. 1f). Lipid accumulation in liver tissues was also significantly $(P<0.05)$ increased in the HF group (Fig. $1 \mathrm{~g}$ and $\mathrm{h}$ ). In addition, HF significantly $(P<0.05)$ increased the contents of hepatic lipids more than NF (Fig. 1i).

To identify the candidate gene products involved in the development of hepatic steatosis, we conducted proteome and transcriptome analyses of these liver tissues. For the 2-DE experiments, we detected 41 spots that were significantly $(P<0.05)$ altered in the HF group (Fig. 2a and Additional file 4: Table S4). These zebrafish 


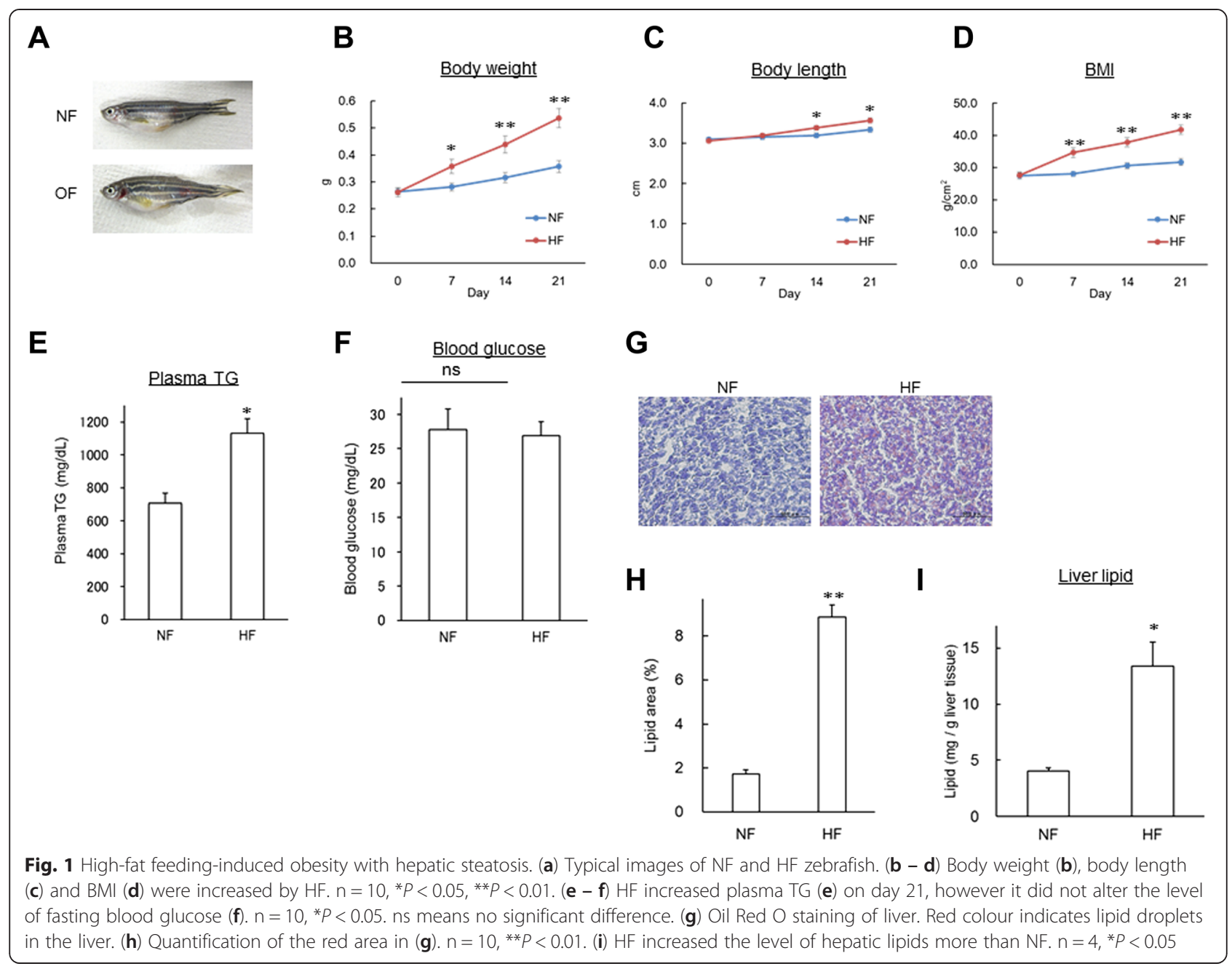

proteins corresponded to 23 human orthologs. Analysis of the genes with altered expression by gene ontology category using GOStat [28] revealed that amino acid metabolism, glucose metabolism, endoplasmic reticulum and mitochondria functions were altered in the $\mathrm{HF}$ group (Additional file 5: Table S5).

Since the 2-DE analysis is limited to the genomic coverage, next we conducted transcriptome studies using DNA microarrays. We found that 125 and 63 human orthologs are significantly $(P<0.01)$ increased and decreased by HF, respectively (Additional file 6: Table S6). Table 2 shows the list of altered genes common to 2-DE and DNA microarray. Of these, fatty acid-binding protein 3 (fabp3) protein and mRNA were significantly $(P<0.05)$ increased in HF (Fig. $2 \mathrm{~b}$ and $\mathrm{c})$. For the liverspecific fabp isoform, fabp $1 b$, there is no significant difference between NF and HF groups (Fig. 2d). Next, we conducted sub-network enrichment analysis (SNEA) [25] with DNA microarray data, which predicts the upstream pathways from the list of altered genes (Additional file 7: Table S7). In this list, we predicted two groups of transcription factors, E2F transcription factors and Kruppel-like factor (KLF) transcription factors, would be involved in the development of the hepatic steatosis. Next, we conducted in silico promoter analysis of zebrafish fabp3 using the JASPAR CORE database [29]. We found that the fabp3 promoter has six E2F-binding sites and two KLF-binding sites, respectively (Fig. 3a). Of the E2F family genes, we found that $e 2 f 3$ and $e 2 f 8$ were significantly $(P<0.05)$ increased in the HF group by qPCR (Fig. $3 \mathrm{~b}$ and c). The expression change of $e 2 f 3$ is consistent with the DNA microarray result. In DNA microarray analysis, e2f4 was also decreased in the HF group (Additional file 6: Table S6), however expression of e2f4 was not detected by qPCR. E2f7 expression was significantly $(P<0.01)$ increased in the DNA microarray experiment (Additional file 6: Table S6), but there was no significant difference between the NF and HF groups (Fig. 3d). 


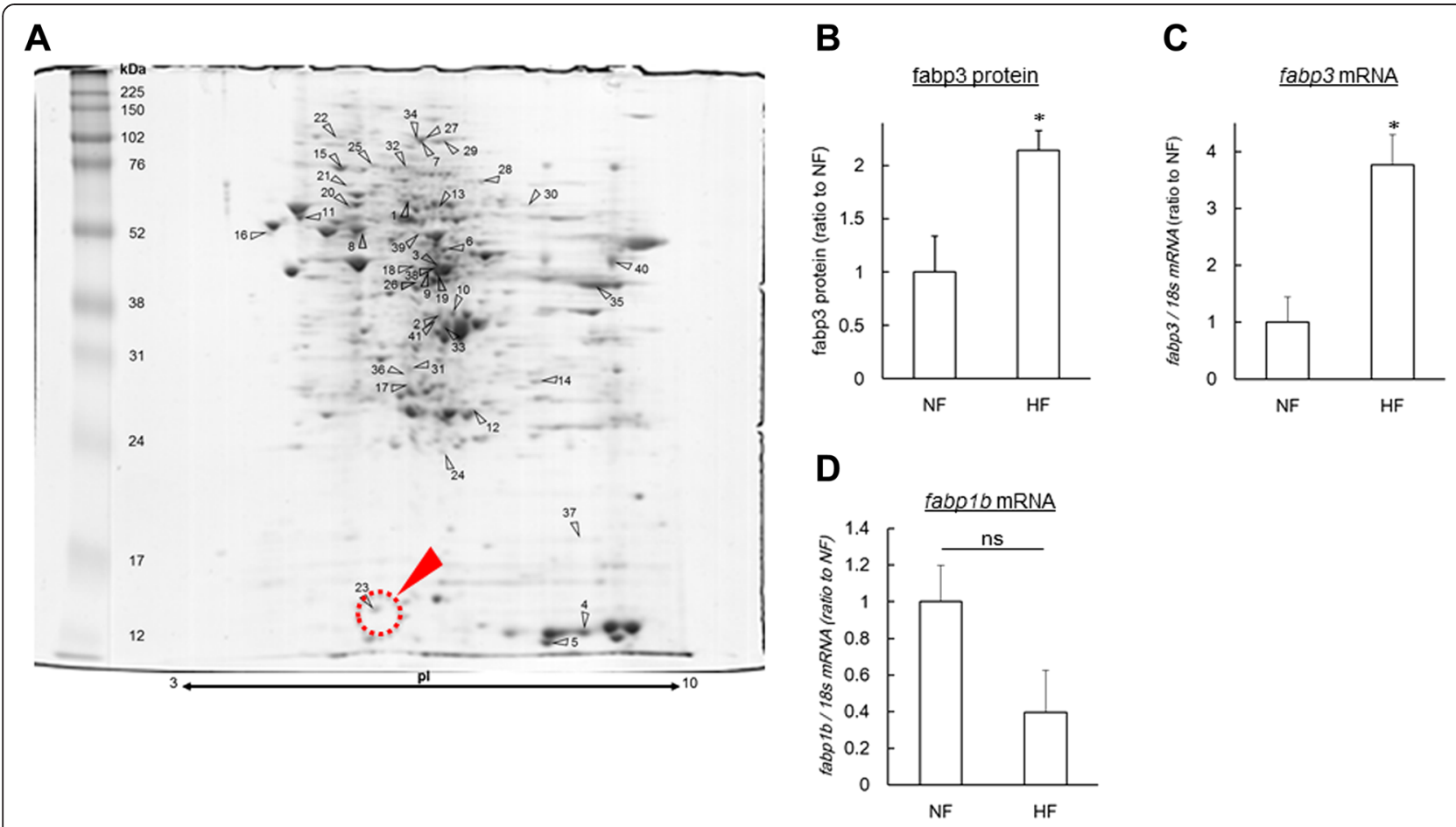

Fig. 2 Fabp3 is increased in liver of DIO-zebrafish. (a) Gel image of 2-DE analysis of DIO-zebrafish liver. The spot numbers in the gel image correspond to Additional file 4: Table S4. Red arrow head indicates the spot of Fabp3 protein. (b - c) Fabp3 protein and fabp3 mRNA expression were measured by 2-DE (b) and qPCR (c). $n=3-5,{ }^{*} P<0.05$. (d) fabp 16 mRNA expression was measured by qPCR. $n=3-5$

\section{Fabp3, e2f3 and E2f8 knockdown suppressed lipid accumulation of liver in DIO-zebrafish}

To examine the contribution of e2f3, e2f8 and fabp3 to the development of hepatic steatosis, we conducted fabp3, e2f3 and e2f8-specific MO (MO-fabp3, MO-e2f3 and $\mathrm{MO}-\mathrm{e} 2 \mathrm{f} 8$ ) i.p. administration every week during feeding experiments of DIO-zebrafish as previously described [26]. There was no appetite suppression by MOs during feeding experiments (data not shown). On day 14, after MO-e2f8 administration three times (starting from 1 week before the feeding experiment), e2f8 protein was reduced to almost $40 \%$ that of control $\mathrm{MO}$ with HF (Fig. 4a). We also tried e2f3 and fabp3 Western blot, but could not succeeded, probably because of the difference of immunogen's sequence between mammals and zebrafish. There was no difference in body weight
(Fig. 4b) and length (Fig. 4c) between these i.p.-administered MOs in DIO-zebrafish. Plasma TG was not affected by these MOs (Fig. 4d), however fasting blood glucose was increased by MO-fabp3 (Fig. 4e). All of these MOs (fabp3, e2f3 and e2f8) reduced lipid accumulation of liver tissues more than HF with control MO, which was detected by Oil Red staining (Fig. 4f).

\section{E2F8 regulates FABP3 expression in zebrafish and HepG2 cells}

In the DIO-zebrafish, MO-e2f8 significantly $(P<0.05)$ suppressed fabp3 mRNA expression in liver (Fig. 5a). The MO-e2f3 showed a trend to suppress fabp3 expression without significance. To examine whether the alterations of fabp3 expression by these MO-i.p. could also be extrapolated to humans, we conducted forced

Table 2 Gene list common to 2-DE and DNA microarray

\begin{tabular}{|c|c|c|c|c|c|}
\hline Protein name & ZF Gene ID & Human ortholog & Human Gene ID & 2-DE & DNA microarray \\
\hline heat shock $70 \mathrm{kDa}$ protein 8 & 573376 & HSPA8 & 3312 & 2.66 & 0.64 \\
\hline valosin containing protein & 327197 & VCP & 7415 & 2.25 & 1.50 \\
\hline fatty acid-binding protein, heart (FABP3) & 171478 & FABP3 & 2170 & 2.15 & 2.49 \\
\hline Adenosylhomocysteinase & 387530 & $\mathrm{AHCY}$ & 191 & 0.49 & 0.66 \\
\hline haemoglobin subunit alpha & 30507 & $\mathrm{HBZ}$ & 3050 & 0.21 & 0.52 \\
\hline
\end{tabular}




\section{A}

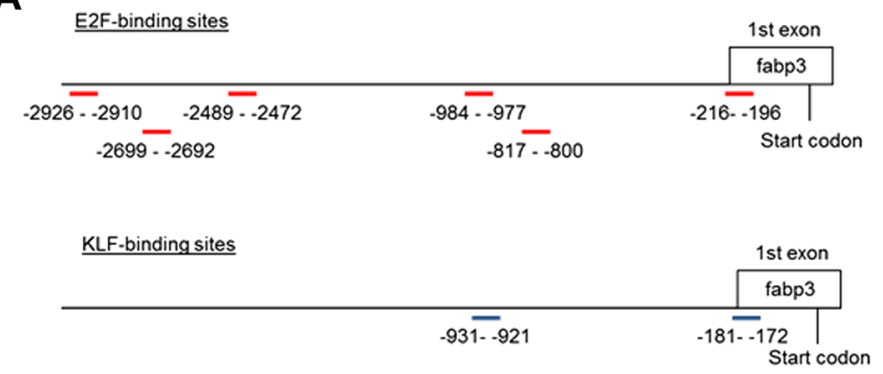

B

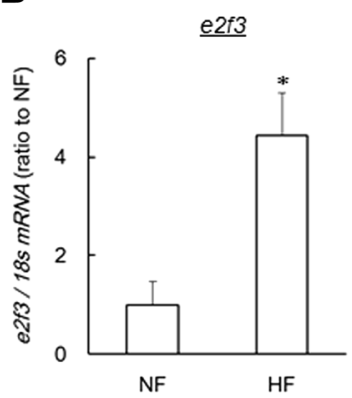

C

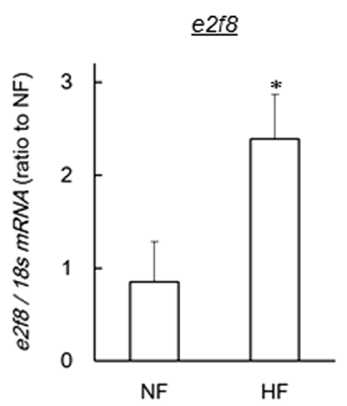

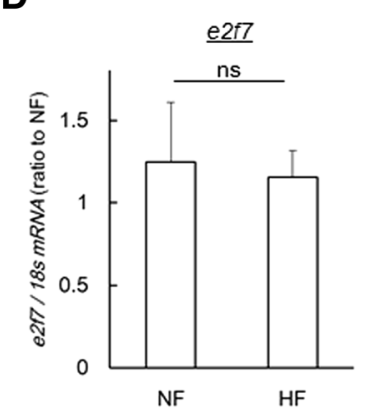

Fig. 3 E2f3 and e2f8 are candidates of fabp3 transactivation in DIO-zebrafish. (a) E2F and KLF-binding site analysis of the zebrafish fabp3 promoter region. Red and blue short bars represent binding sites of E2F and KLF, respectively. Numbers indicate the $5^{\prime}$ location of the binding site from the start codon of each gene. (b-d) E2f family gene expression in DIO-zebrafish. HF increased e2f3 (b) and e2f8 (c) expression, not e2f7 (d). $n=4,{ }^{*} P<0.05$

expression of E2F3 and E2F8 in HepG2 human hepatocarcinoma cells (Fig. 5b and c). E2F8, not E2F3, overexpression increased $F A B P 3$ expression significantly $(P<0.05$; Fig. $5 \mathrm{~d})$. Other FABP family, $F A B P 1, F A B P 5$ and $F A B P 7$ expression levels were not altered by $E 2 F 3$ or E2F8 overexpression (Fig. $5 \mathrm{e}-\mathrm{g}$ ). These results indicates that E2F8 could positively regulate $F A B P 3$ expression in human and zebrafish.

\section{Co-administration of anserine and creatine suppressed obesity-associated phenotypes via downregulation of fabp3}

To confirm that fabp3 expression is responsible for the improvement of hepatic steatosis, we administered anserine and creatine to DIO-zebrafish as anti-obesity therapeutics. Anserine and creatine are known to alter insulin secretion and glycogen metabolism, changes that ameliorate the obesity phenotype in a rodent model of obesity [30, 31] and in human clinical trials [32]. We fed anserine and creatine-containing food (AC) to DIOzebrafish for 21 days. AC significantly $(P<0.05)$ suppressed the increase in body weight (Fig. 6a) without an effect on body length (Fig. 6b) or on feeding volume (Fig. 6c). Although AC could not suppress the increase in plasma TG (Fig. 6d), AC suppressed the increase in fasting blood glucose significantly $(P<0.05$ vs. HF;
Fig. 6e), similar to the previous study in rodent models $[30,31]$. AC also suppressed the accumulation of lipid droplets in liver tissues $(P<0.01$, Fig. 6f). qPCR analysis of the liver tissues revealed that the increases in fabp3, $e 2 f 8$ and $f a b p 3$ expression were also suppressed by $\mathrm{AC}$ administration $(P<0.05$ vs. HF, Fig. $7 \mathrm{a}-\mathrm{c})$ in the liver.

\section{Discussion}

\section{FABP3 involvement in hepatic steatosis}

FABP family members are in charge of regulating fatty acid uptake and intracellular transport. Zebrafish have seven fabp family genes, which correspond to mammalian FABP1, FABP2, FABP3, FABP6, FABP7, FABP10 and FABP11. FABP3 regulates metabolic homeostasis and developmental regulation by modulating intracellular lipid transport in cardiac muscles of human [33] and zebrafish [34, 35]. FABP3 mRNA has been shown to be markedly up-regulated in liver and adipose tissue in rats with high-cholesterol diet [36, 37]. Furthermore, hesperidin, a citrus bioflavonoid, ameliorated liver steatosis in high-cholesterol diet rats through FABP3 down regulation in the liver [37]. In the Gene Expression Omnibus (GEO) [38], FABP3 was significantly $(P<0.05)$ increased in mouse livers with a ketogenic diet (GDS2738 [39]). The ketogenic diet is "high-fat, low carbohydrate", similar to the HF group in our zebrafish experiments. In 


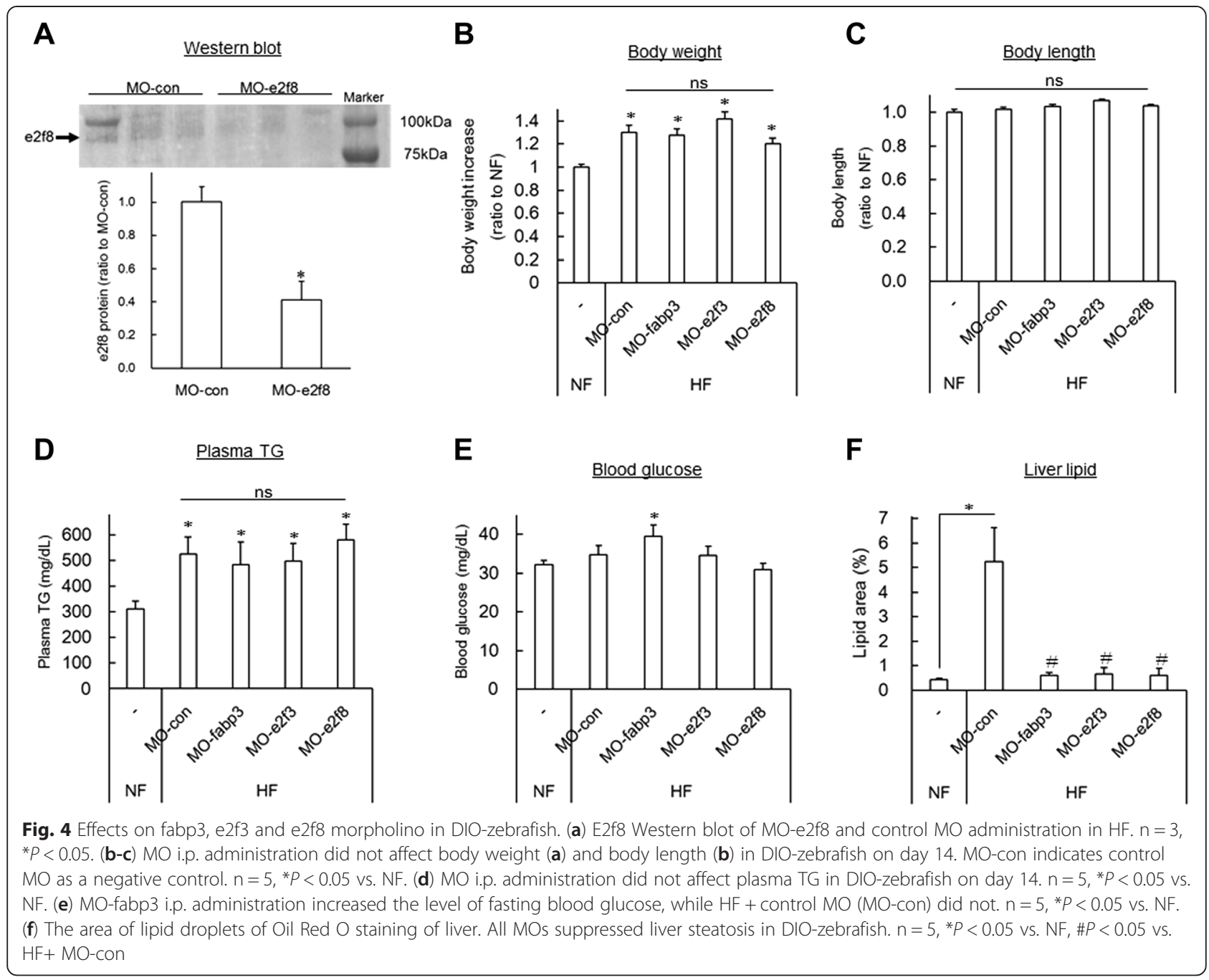

human, Başar $\mathrm{O}$ et al. reported increased human FABP3 concentrations in non-alcoholic fatty liver disease [40], metabolic disease [41] and in conditions of impaired glucose metabolism [42]. In zebrafish, fabp3 is expressed in the liver of adult fish [43] and was induced by highlinoleic acid-rich foods [44]. In contrast to fabp3, FABP1, a liver-type FABP, is increased in hepatic steatosis in mammals and has a protective role against lipid toxicity [9]. The zebrafish homolog of human FABP1, fabplb, is required for liver development [45]; however, the relationship between fabp1b and hepatic steatosis has not been reported. In our study, zebrafish fabplb shows a decreasing trend in the HF group without significance (Fig. 2d), partially consistent with the down regulation in hepatic steatosis in mammals. Unlike mammals, it is noteworthy that fabp3 is expressed in liver, not in the heart of adult zebrafish [43], suggesting the possibility that fabp3 shares a function in lipid metabolism with fabp1b.

\section{The E2F8-FABP3 pathway promotes hepatic steatosis}

In the current study, SNEA and promoter analysis predicted the involvement of the E2F family in hepatic steatosis. E2F family members play a major role during the G1/S transition of the cell cycle across phyla from plants to mammals. The E2F family is generally split by function into two groups: transcriptional activators (E2F1, $\mathrm{E} 2 \mathrm{~F} 2$ and E2F3a) and transcriptional repressors (E2F3b, E2F4, E2F5, E2F6, E2F7 and E2F8) [46]. However, recent studies of mouse knockout tissues demonstrate that E2F function in vivo does not strictly adhere to this elegant dichotomous paradigm [47]. E2Fs also regulate the expression of the metabolic genes involved in hepatic steatosis [48] and obesity [49]. There is a limited number of studies related to E2F8 and obesity, however, these are still controversial. E2F8 expression was increased (about 7-fold) in the adipose tissue of DIO-mice [50], similar to our result. Partial deficiency in the retinoblastoma protein gene, which is upstream of E2F8 [51], protects 


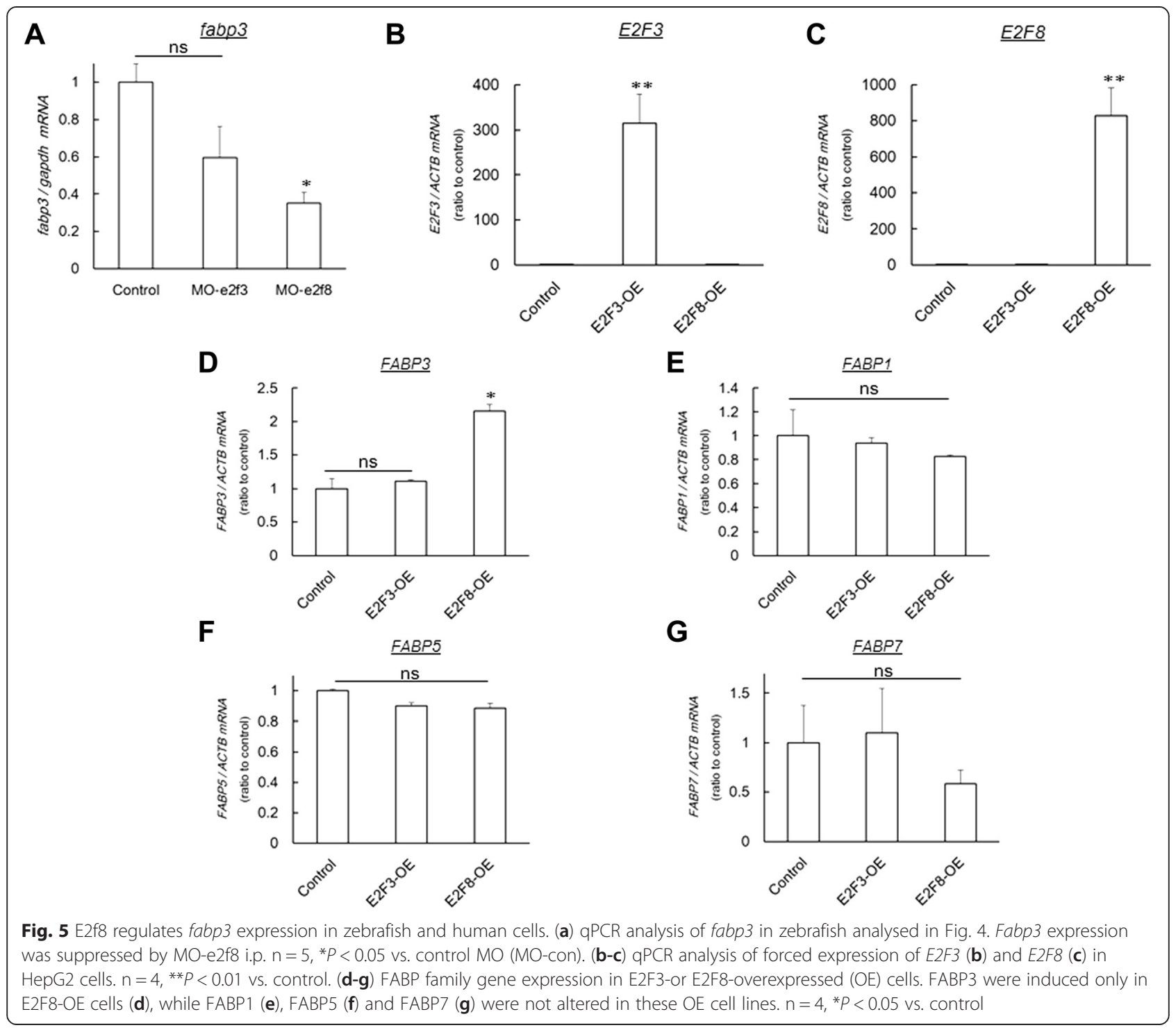

against the development of obesity and associated metabolic disturbances [52], also consistent with our results. Peroxisome proliferator-activated receptor $\beta$ (PPAR $\beta$ ) knockout (KO) suppressed E2F8 mRNA expression in liver regeneration in mice [48]. Since activation of the PPAR $\beta / \delta$ complex inhibits hepatic steatosis [53], PPAR $\beta$ KO-induced E2F8 suppression seems to worsen hepatic steatosis, which seems to be contrary to our result with MO-e2f8. However, with anti-obese compounds, AC suppressed hepatic steatosis, consistent with mammals, through suppression of $e 2 f 3, e 2 f 8$ and fabp3. Additionally, anserine and creatine administration to DIOzebrafish ameliorated the hepatic steatosis with down regulation of e2f8 and fabp3 in the current study. Creatine normalizes PPAR $\alpha$ downregulation in HF-rats [54], and PPAR $\alpha$ agonist induced FABP3 gene expression [55].
Thus we hypothesized that E2F8 transcription factor mediated FABP3 transactivation might be a downstream of PPAR $\alpha$ in the development of hepatic steatosis. These results strengthened the notion that the E2F-FABP3 pathway is involved in hepatic steatosis. E2f3 was also increased in the liver of DIO-zebrafish, and MO-E2F3 ameliorated the phenotype independent of fabp3 expression. While no studies have examined the relationship between E2F3 and hepatic steatosis, E2F3 was found to be increased in the liver of HF-induced hepatic steatosis of mice (GDS4013 [56]) and clinical alcohol-induced hepatitis (GDS4389 [57]) in GEO. These results indicate that E2F3 would also be a strong candidate to promote hepatic steatosis. In fact, Asp P et al. found that mouse E2f3b, a shorter isoform of E2f3, bind a large number of lipid metabolism genes in myogenic differentiation [58], suggesting 


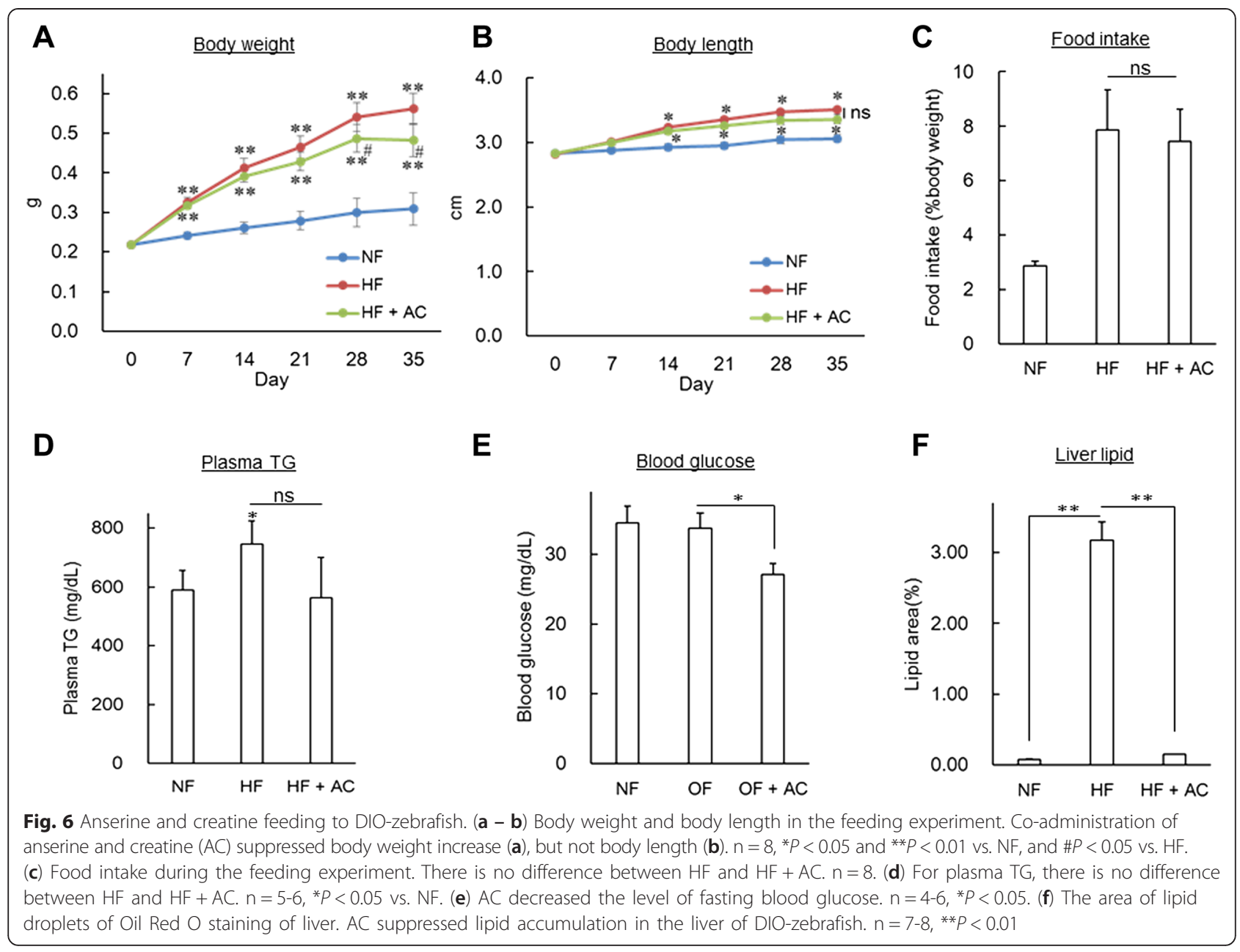

that E2F3 also might be involved in hepatic steatosis which is independent to FABP3. The actual DNA-binding affinity of E2F8 to FABP3 promoter region and its transactivation will be elucidated by ChIP analysis. Additionally, to confirm the common function of the E2F8-FABP3 pathway between zebrafish and mammals in hepatic steatosis, in vitro and mammalian model experiments should be completed in future.

\section{Conclusions}

Using proteome and transcriptome analysis in DIOzebrafish, we discovered that the E2F8-FABP3 pathway
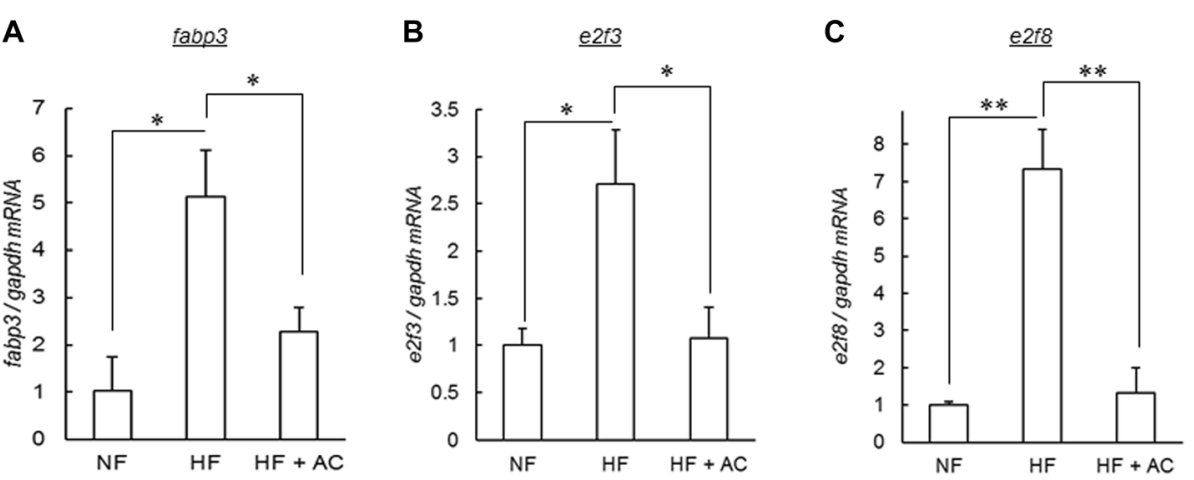

Fig. 7 Anserine and creatine effects on fabp3, e2f3 and e2f8 expression. (a-c) qPCR analysis of AC-administration in DIO-zebrafish. Fabp3 (a), e2f3 (b) and e2f8 mRNA (c) were increased by HF, and then decreased by $A C$ ( $H F+A C$ group). $n=4$, ${ }^{*} P<0.05$, ${ }^{*} P<0.01$ 
is one of the contributing factors to promoting hepatic steatosis in DIO-zebrafish. Combination analysis of the in vivo, in vitro and in silico data will hopefully lead to this pathway being a therapeutic target against dietinduced hepatic steatosis.

\section{Additional files}

Additional file 1: Table S1. Fatty acid composition of fish foods.
Additional file 2: Table S2. MO sequences.
Additional file 3: Table S3. Primer sequences for QPCR.
Additional file 4: Table S4. Protein list altered in DIO-zebrafish.
Additional file 5: Table S5. Gene ontology analysis of proteins altered
in DIO-zebrafish.
Additional file 6: Table S6. Gene list altered in DIO-zebrafish.
Additional file 7: Table S7. SNEA analysis.

\section{Abbreviations}

2-DE: Two-dimensional electrophoresis; AC: Anserine and creatine-containing food; BMI: Body mass index; DIO-zebrafish: Diet-induced obesity zebrafish; DTT: Dithiothreitol; E2F: E2F transcription factor; FABP: Fatty acid binding protein; GEO: Gene expression omnibus; HL450: Hikari Labo M-450; HL450BT: Hikari Labo M-450 containing beef tallow; i.p.: Intraperitoneal injection; KLF: Kruppel-like factor; MO: Morpholino antisense oligonucleotides; mpf: Months post fertilization; NAFLD: Non-alcoholic fatty liver disease; NASH: Non-alcoholic steatohepatitis; NF: Normal feeding; PPAR: Peroxisome proliferator-activated receptor; SNEA: Sub-network enrichment analysis; TG: Triacylglyceride.

\section{Competing interests}

The authors declare that they have no competing interests.

\section{Authors' contributions}

Conceived and designed the experiments: $H E, T$. Performed the experiments: YS, SK, MA, BZ, NU. Analysed the data: YS, SK, YN. Contributed reagents/materials/ analysis tools: YS2, YT. Wrote the paper: YS, SK. All authors read and approved the final manuscript.

\section{Authors' information}

Yasuhito Shimada, MD, PhD, is an assistant professor at the Department of Molecular and Cellular Pharmacology, Pharmacogenomics and Pharmacoinformatics, Graduate School of Medicine, Mie University. The focus of Dr. Shimada's research is studying obesity prevention using a diet-induced obesity model of zebrafish. Shisei Kuninaga, Yasuhiko Shiina and Yoshinori Takahashi, PhD, are researchers at Maruha Nichiro Corporation. The focus of their research is to create functional foods to combat human obesity. Michiko Ariyoshi is a graduate student at the Department of Molecular and Cellular Pharmacology, Pharmacogenomics and Pharmacoinformatics, Graduate School of Medicine, Mie University. The focus of Ms. Ariyoshi's research is studying obesity prevention using a diet-induced obesity model of zebrafish. Beibei Zhang, PhD, was a graduate student at the Department of Molecular and Cellular Pharmacology, Pharmacogenomics and Pharmacoinformatics, Graduate School of Medicine, Mie University. The focus of Dr. Zhang's research is studying the prevention of obesity using a diet-induced obesity model of zebrafish. Noriko Umemoto, PhD, was a graduate student in the Department of Molecular and Cellular Pharmacology, Pharmacogenomics and Pharmacoinformatics, Graduate School of Medicine, Mie University. The focus of Dr. Umemoto's research was studying the prevention of obesity using a diet-induced obesity model of zebrafish. Yuhei Nishimura, MD, PhD, is a lecturer in the Department of Molecular and Cellular Pharmacology, Pharmacogenomics and Pharmacoinformatics, Graduate School of Medicine, Mie University. Dr Nishimura's research interests are bioinformatics and pharmacoinformatics. Hiroyuki Enari is a project manager of Maruha Nichiro Corporation. Mr. Enari's research interests are fisheries biotechnology and food science. Toshio Tanaka, MD, PhD, is a professor in the Department of Molecular and Cellular Pharmacology, Pharmacogenomics and Pharmacoinformatics,
Graduate School of Medicine, Mie University. The focus of Prof. Tanaka's research is to identify drug targets using a pharmacogenomics approach. Prof. Tanaka has published over 100 peer-reviewed research articles and reviews in international journals.

\section{Acknowledgements}

We would like to thank S. Ichikawa for zebrafish breeding, M. Nakachi for proteome experiments and R. Ikeyama for secretarial assistance.

\section{Author details}

'Department of Molecular and Cellular Pharmacology, Pharmacogenomics and Pharmacoinformatics, Mie University Graduate School of Medicine, 2-174, Edobashi, Tsu, Mie, Japan. ${ }^{2}$ Department of Systems Pharmacology, Mie University Graduate School of Medicine, Mie, Japan. ${ }^{3}$ Mie University Medical Zebrafish Research Center, Mie, Japan. ${ }^{4}$ Department of Bioinformatics, Mie University Life Science Research Center, Mie, Japan. ${ }^{5}$ Department of Omics Medicine, Mie University Industrial Technology Innovation, Mie, Japan.

${ }^{6}$ Central Research Institute, Maruha Nichiro Corporation, Ibaraki, Japan.

Received: 1 December 2014 Accepted: 4 May 2015

Published online: 20 May 2015

\section{References}

1. Angulo P, Lindor KD. Non-alcoholic fatty liver disease. J Gastroenterol Hepatol. 2002;17(Suppl):S186-90

2. Marrero JA, Fontana RJ, Su GL, Conjeevaram HS, Emick DM, Lok AS. NAFLD may be a common underlying liver disease in patients with hepatocellular carcinoma in the United States. Hepatology. 2002;36:1349-54.

3. Pagano G, Pacini G, Musso G, Gambino R, Mecca F, Depetris N, et al. Nonalcoholic steatohepatitis, insulin resistance, and metabolic syndrome: further evidence for an etiologic association. Hepatology. 2002;35:367-72

4. Moore JB. Non-alcoholic fatty liver disease: the hepatic consequence of obesity and the metabolic syndrome. Proc Nutr Soc. 2010;69:211-20.

5. McCullough AJ. Update on nonalcoholic fatty liver disease. I Clin Gastroenterol. 2002;34:255-62.

6. Sanyal AJ, Campbell-Sargent C, Mirshahi F, Rizzo WB, Contos MJ, Sterling RK, et al. Nonalcoholic steatohepatitis: association of insulin resistance and mitochondrial abnormalities. Gastroenterology. 2001;120:1183-92.

7. Neuschwander-Tetri BA. Hepatic lipotoxicity and the pathogenesis of nonalcoholic steatohepatitis: the central role of nontriglyceride fatty acid metabolites. Hepatology. 2010;52:774-88.

8. Berlanga A, Guiu-Jurado E, Porras JA, Auguet T. Molecular pathways in nonalcoholic fatty liver disease. Clin Exp Gastroenterol. 2014;7:221-39.

9. Thumser AE, Moore JB, Plant NJ. Fatty acid binding proteins: tissue-specific functions in health and disease. Curr Opin Clin Nutr Metab Care. 2014;17:124-9.

10. Lieschke G, Currie P. Animal models of human disease: zebrafish swim into view. Nat Rev Genet. 2007;8:353-67.

11. Shimada Y, Hirano M, Nishimura Y, Tanaka T. A high-throughput fluorescence-based assay system for appetite-regulating gene and drug screening. PLoS One. 2012;7, e52549.

12. Henderson RJ, Tocher DR. The lipid composition and biochemistry of freshwater fish. Prog Lipid Res. 1987;26:281-347.

13. Hölttä-Vuori M, Salo VT, Nyberg L, Brackmann C, Enejder A, Panula P, et al. Zebrafish: gaining popularity in lipid research. Biochem J. 2010;429:235-42.

14. Song $\mathrm{Y}$, Cone RD. Creation of a genetic model of obesity in a teleost. FASEB J. 2007:21:2042-9.

15. Oka T, Nishimura Y, Zang L, Hirano M, Shimada Y, Wang Z, et al. Dietinduced obesity in zebrafish shares common pathophysiological pathways with mammalian obesity. BMC Physiol. 2010;10:21.

16. Tainaka T, Shimada Y, Kuroyanagi J, Zang L, Oka T, Nishimura Y, et al. Transcriptome analysis of anti-fatty liver action by Campari tomato using a zebrafish diet-induced obesity model. Nutr Metab (Lond). 2011;8:88.

17. Stoletov K, Fang L, Choi SH, Hartvigsen K, Hansen LF, Hall C, et al. Vascular lipid accumulation, lipoprotein oxidation, and macrophage lipid uptake in hypercholesterolemic zebrafish. Circ Res. 2009;104:952-60.

18. Hiramitsu M, Shimada Y, Kuroyanagi J, Inoue T, Katagiri T, Zang L, et al. Eriocitrin ameliorates diet-induced hepatic steatosis with activation of mitochondrial biogenesis. Sci Rep. 2014;4:3708. 
19. Westerfield M. The zebrafish book: A guide for the labortory use of zebrafish danio (branchydanio) rerio. 5th ed. Eugene: University of Oregon press; 2007.

20. Zang L, Morikane D, Shimada Y, Tanaka T, Nishimura N. A novel protocol for the oral administration of test chemicals to adult zebrafish. Zebrafish. 2011;8:203-10

21. Gonzales Jr JM. Preliminary evaluation on the effects of feeds on the growth and early reproductive performance of zebrafish (Danio rerio). J Am Assoc Lab Anim Sci. 2012;51:412-7.

22. Zang $L$, Shimada $Y$, Nishimura $Y$, Tanaka T, Nishimura N. A novel, reliable method for repeated blood collection from aquarium fish. Zebrafish. 2013:"10(3):425-32

23. Zang L, Shimada Y, Kawajiri J, Tanaka T, Nishimura N. Effects of Yuzu (Citrus junos Siebold ex Tanaka) peel on the diet-induced obesity in a zebrafish model. J Funct Foods. 2014;10:499-510.

24. Folch J, Lees M, Sloane Stanley GH. A simple method for the isolation and purification of total lipides from animal tissues. J Biol Chem. 1957:226:497-509.

25. Kotelnikova E, Yuryev A, Mazo I, Daraselia N. Computational approaches for drug repositioning and combination therapy design. J Bioinform Comput Biol. 2010;8:593-606.

26. Shimada $Y$, Kuroyanagi J, Zhang B, Ariyoshi M, Umemoto N, Nishimura $Y$, et al. Downregulation of max dimerization protein 3 is involved in decreased visceral adipose tissue by inhibiting adipocyte differentiation in zebrafish and mice. Int J Obes (Lond). 2013;38:1053-60.

27. Hasumura T, Shimada Y, Kuroyanagi J, Nishimura Y, Meguro S, Takema Y, et al. Green tea extract suppresses adiposity and affects the expression of lipid metabolism genes in dietinduced obese zebrafish. Nutr Metab (Lond). 2012;9:73.

28. Ashburner M, Ball CA, Blake JA, Botstein D, Butler H, Cherry JM, et al. Gene ontology: tool for the unification of biology. The Gene Ontology Consortium. Nat Genet. 2000;25:25-9

29. Wasserman WW, Sandelin A. Applied bioinformatics for the identification of regulatory elements. Nat Rev Genet. 2004;5:276-87.

30. Takahashi $Y$, Kawarasaki M, Hoshino $Y$, Honmura A, Enari H. The suppressive effect of salmon muscle extracts containing anserine on lipid accumulation in rats fed with high fat diet. Nippon Suisan Gakkaishi. 2008;74:1075-81.

31. Rooney K, Bryson J, Phuyal J, Denyer G, Caterson I, Thompson C. Creatine supplementation alters insulin secretion and glucose homeostasis in vivo. Metabolism. 2002:51:518-22.

32. Field AE, Austin SB, Camargo CA, Taylor CB, Striegel-Moore RH, Loud KJ, et al. Exposure to the mass media, body shape concerns, and use of supplements to improve weight and shape among male and female adolescents. Pediatrics. 2005;116:e214-20.

33. Binas B, Danneberg $H$, McWhir J, Mullins L, Clark AJ. Requirement for the heart-type fatty acid binding protein in cardiac fatty acid utilization. FASEB J. 1999:13:805-12.

34. Wang $X$, Zhou L, Jin J, Yang $Y$, Song G, Shen $Y$, et al. Knockdown of FABP3 impairs cardiac development in zebrafish through the retinoic acid signaling pathway. Int J Mol Sci. 2013;14:13826-41.

35. Liu YQ, Song GX, Liu HL, Wang XJ, Shen YH, Zhou LJ, et al. Silencing of FABP3 leads to apoptosis-induced mitochondrial dysfunction and stimulates Wnt signaling in zebrafish. Mol Med Rep. 2013;8:806-12.

36. Matsuda A, Wang Z, Takahashi S, Tokuda T, Miura N, Hasegawa J. Upregulation of mRNA of retinoid binding protein and fatty acid binding protein by cholesterol enriched-diet and effect of ginger on lipid metabolism. Life Sci. 2009:84:903-7.

37. Wang $X$, Hasegawa J, Kitamura Y, Wang Z, Matsuda A, Shinoda W, et al. Effects of hesperidin on the progression of hypercholesterolemia and fatty liver induced by high-cholesterol diet in rats. J Pharmacol Sci. 2011;117:129-38.

38. Edgar R, Domrachev M, Lash AE. Gene Expression Omnibus: NCBI gene expression and hybridization array data repository. Nucleic Acids Res. 2002;30:207-10.

39. Kennedy AR, Pissios P, Otu H, Roberson R, Xue B, Asakura K, et al. A high-fat, ketogenic diet induces a unique metabolic state in mice. Am J Physiol Endocrinol Metab. 2007;292:E1724-39.

40. Başar O, Akbal E, Köklü S, Tuna Y, Koçak E, Başar N, et al. Increased H-FABP concentrations in nonalcoholic fatty liver disease. Possible marker for subclinical myocardial damage and subclinical atherosclerosis. Herz. 2013;38:417-22
41. Akbal E, Ozbek M, Gunes F, Akyurek O, Ureten K, Delibasi T. Serum heart type fatty acid binding protein levels in metabolic syndrome. Endocrine. 2009;36:433-7.

42. Karbek B, Özbek M, Bozkurt NC, Ginis Z, Güngünes A, Ünsal I, et al. Hearttype fatty acid binding protein (H-FABP): relationship with arterial intima-media thickness and role as diagnostic marker for atherosclerosis in patients with Impaired glucose metabolism. Cardiovasc Diabetol. 2011;10:37.

43. Liu RZ, Denovan-Wright EM, Wright JM. Structure, linkage mapping and expression of the heart-type fatty acid-binding protein gene (fabp3) from zebrafish (Danio rerio). Eur J Biochem. 2003;270:3223-34.

44. Venkatachalam AB, Sawler DL, Wright JM. Tissue-specific transcriptional modulation of fatty acid-binding protein genes, fabp2, fabp3 and fabp6, by fatty acids and the peroxisome proliferator, clofibrate, in zebrafish (Danio rerio). Gene. 2013;520:14-21.

45. Her GM, Chiang CC, Chen WY, Wu JL. In vivo studies of liver-type fatty acid binding protein (L-FABP) gene expression in liver of transgenic zebrafish (Danio rerio). FEBS Lett. 2003;538:125-33.

46. Harbour JW, Dean DC. The Rb/E2F pathway: expanding roles and emerging paradigms. Genes Dev. 2000;14:2393-409.

47. Chen HZ, Ouseph MM, Li J, Pecot T, Chokshi V, Kent L, et al. Canonical and atypical E2Fs regulate the mammalian endocycle. Nat Cell Biol. 2012;14:1192-202.

48. Liu HX, Fang Y, Hu Y, Gonzalez FJ, Fang J, Wan YJ. PPARß Regulates Liver Regeneration by Modulating Akt and E2f Signaling. PLoS One. 2013;8, e65644.

49. Blanchet E, Annicotte JS, Lagarrigue S, Aguilar V, Clapé C, Chavey C, et al. E2F transcription factor-1 regulates oxidative metabolism. Nat Cell Biol. 2011;13:1146-52.

50. Suwa A, Yoshino M, Yamazaki C, Naitou M, Fujikawa R, Matsumoto S, et al. RMI1 deficiency in mice protects from diet and genetic-induced obesity. FEBS J. 2010:277:677-86.

51. Christensen J, Cloos P, Toftegaard U, Klinkenberg D, Bracken AP, Trinh E, et al. Characterization of E2F8, a novel E2F-like cell-cycle regulated repressor of E2F-activated transcription. Nucleic Acids Res. 2005;33:5458-70.

52. Mercader J, Ribot J, Murano I, Feddersen S, Cinti S, Madsen L, et al. Haploinsufficiency of the retinoblastoma protein gene reduces diet-induced obesity, insulin resistance, and hepatosteatosis in mice. Am J Physiol Endocrinol Metab. 2009;297:E184-93.

53. Qin X, Xie X, Fan Y, Tian J, Guan Y, Wang X, et al. Peroxisome proliferatoractivated receptor-delta induces insulin-induced gene-1 and suppresses hepatic lipogenesis in obese diabetic mice. Hepatology. 2008;48:432-41.

54. Deminice R, Da Silva RP, Lamarre SG, Brown C, Furey GN, McCarter SA, et al. Creatine supplementation prevents the accumulation of fat in the livers of rats fed a high-fat diet. J Nutr. 2011;141:1799-804.

55. Hyder A, Zenhom M, Klapper M, Herrmann J, Schrezenmeir J. Expression of fatty acid binding proteins 3 and 5 genes in rat pancreatic islets and INS-1E cells: regulation by fatty acids and glucose. Islets. 2010;2:174-84.

56. Duval C, Thissen U, Keshtkar S, Accart B, Stienstra R, Boekschoten MV, et al. Adipose tissue dysfunction signals progression of hepatic steatosis towards nonalcoholic steatohepatitis in C57BL/6 mice. Diabetes. 2010;59:3181-91.

57. Affò S, Dominguez M, Lozano JJ, Sancho-Bru P, Rodrigo-Torres D, Morales-Ibanez O, et al. Transcriptome analysis identifies TNF superfamily receptors as potential therapeutic targets in alcoholic hepatitis. Gut. 2013;62:452-60

58. Asp P, Acosta-Alvear D, Tsikitis M, Van Oevelen C, Dynlacht BD. E2f3b plays an essential role in myogenic differentiation through isoform-specific gene regulation. Genes Dev. 2009;23:37-53. 